Journal of

Vibration

and Acoustics

\title{
Technical Briefs
}

\section{Stability Analysis of a Single-Ball Automatic Balancer}

\section{Chung-Jen Lu}

Associate Professor

Department of Mechanical Engineering,

National Taiwan University,

No. 1 Roosevelt Rd. Sec. 4,

Taipei 10617, Taiwan, R.O.C.

e-mail: cjlu@ntu.edu.tw

Under proper working conditions, a ball-type automatic balancer can effectively reduce the imbalance vibrations of an optical disk drive. The proper working conditions can be determined by a stability analysis of the equilibrium states of the nonlinear system formed by the rotating disk, balancer, and suspension system. Several attempts have been made to study the stability of the equilibrium states numerically in some finite regions of the relevant parameter space. This paper in contrast analytically investigates the stability characteristics of the equilibrium states. A theoretical model of an optical disk drive packed with an automatic balancer is constructed first. The governing equations of the theoretical model are derived using Lagrange's equations and closed-form formulas for the equilibrium positions are presented. Finally, general guidelines on the stability of the equilibrium states are proposed. [DOI: 10.1115/1.2149398]

Keywords: automatic balancer, stability, vibration, optical disk

\section{Introduction}

The eccentric mass of an optical disk can cause serious vibrations at high rotating speeds. Since the amount of eccentricity changes from disk to disk, it is desirable to equip an optical disk drive with an automatic balancer system (ABS) that can automatically eliminate the unbalance associated with each disk. The most popular type of ABS's in use in the optical disk drive industry is the ball-type that is characterized by having several balls moving freely on a single or several circular orbits. Under proper working conditions, the balls will move to the equilibrium positions or states where vibrations due to unbalance are totally suppressed. These equilibrium positions are called the perfect balancing positions. The stability characteristics of the perfect balancing positions are closely related to the proper working conditions [1-4] and have been investigated numerically [5-7]. Specifically, [5-7]

Contributed by the Technical Committee on Vibration and Sound of ASME for publication in the Journal of Vibration and Acoustics. Manuscript received August 5, 2004; final manuscript received September 7, 2005. Assoc. Editor: Joseph P. Cusumano. employed two design parameters, which formed a parameter plane, and numerically determined the stable region of each equilibrium state on the parameter plane. Such numerical analyses make it difficult to draw general guidelines on the stability of the equilibrium states.

In this paper we analytically study the stability of each equilibrium state of a rotating disk equipped with a single-ball ABS. First the nonlinear equations of motion are derived with respect to a co-rotating coordinates system. Then equilibrium states and the associated linearized equations are obtained. Finally the conditions under which each equilibrium state is unstable are determined analytically by Routh's criterion and the results summarized.

\section{Mathematical Model and Governing Equations}

Figure 1 depicts schematically the system considered, which consists of the following components:

- A rotating unbalanced disk with mass $m_{d}$ and a constant angular speed $\omega$. The mass center $G$ of the disk is located at a distance $e$ from the geometric center $C$.

- An ABS, which is composed of an annular groove containing one or more balls and a damping fluid. The balls can move freely along the groove and their movements are subject to viscous damping only. For the sake of simplicity, we consider here only a single ball and treat the ball as a point mass. Let $d$ denote the radius of the groove, $c_{b}$ the viscous damping factor of the damping fluid, and $m_{b}$ the mass of the ball.

- A suspension system. The flexibility of the suspension system is characterized by equivalent linear springs and viscous dampers, denoted by $\left(k_{x}, k_{y}\right)$ and $\left(c_{x}, c_{y}\right)$, respectively. For simplicity, we assume that $k_{x}=k_{y}=k$, and $c_{x}=c_{y}=c$.

The coordinates system used is shown in Fig. 2. The $x y$-reference frame rotates about a fixed point $O$ with the same angular speed $\omega$ as the disk. When the disk is at rest, the center of the disk $C$ coincides with $O$ at which the suspension springs are undeformed. The rotating $x y$-frame is used because the equations of motion expressed in this frame are autonomous. The rotating disk undergoes a translational motion in the $x y$-frame and its position is indicated by the coordinates $(\hat{x}, \hat{y})$ of the geometric center $C$. The position of the ball is given by the angle $\beta$ relative to the mass center $G$.

The equations of motion are derived from Lagrange's equations and can be expressed in dimensionless form as:

$$
\begin{aligned}
x^{\prime \prime}- & \left(\mu_{b} \sin \beta\right) \beta^{\prime \prime}-\mu_{b}\left(\beta^{\prime}+\Omega\right)^{2} \cos \beta+2 \zeta x^{\prime}-2 \Omega y^{\prime}+\left(1-\Omega^{2}\right) x \\
& -2 \zeta \Omega y^{\prime}-\mu_{b} \Omega^{2} / \eta=0 \\
y^{\prime \prime}+ & \left(\mu_{b} \cos \beta\right) \beta^{\prime \prime}-\mu_{b}\left(\beta^{\prime}+\Omega\right)^{2} \sin \beta+2 \Omega x^{\prime}+2 \zeta y^{\prime}+\left(1-\Omega^{2}\right) y \\
& +2 \zeta \Omega x=0
\end{aligned}
$$




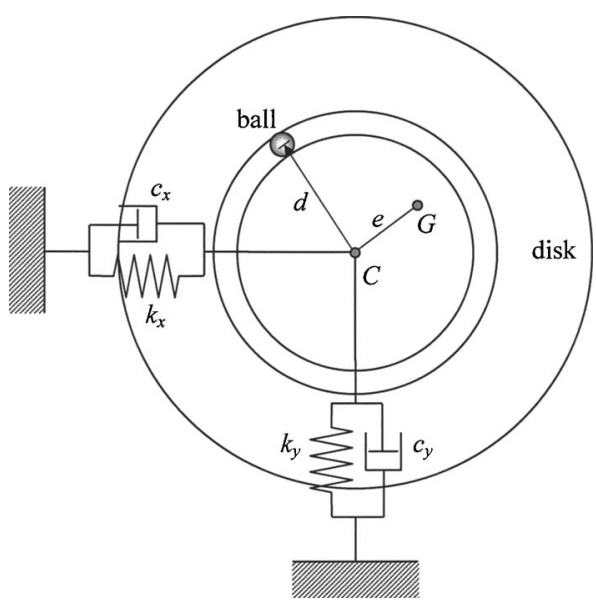

Fig. 1 Schematic diagram of the ball-type automatic balancer, rotating disk and equivalent suspension system

$$
\begin{aligned}
& -\left(\mu_{b} \sin \beta\right) x^{\prime \prime}+\left(\mu_{b} \cos \beta\right) y^{\prime \prime}+\mu_{b} \beta^{\prime \prime}+\left(2 \mu_{b} \Omega \cos \beta\right) x^{\prime} \\
& \quad+\left(2 \mu_{b} \Omega \sin \beta\right) y^{\prime}+2 \zeta_{b} \mu_{b} \beta^{\prime}+\mu_{b} \Omega^{2}(x \sin \beta-y \cos \beta)=0
\end{aligned}
$$

where

$$
\begin{gathered}
x=\hat{x} / d, \quad y=\hat{y} / d, \quad \Omega=\omega / \omega_{n}, \\
M=m_{d}+m_{b}, \quad \omega_{n}=\sqrt{k / M}, \\
\zeta=c /\left(2 M \omega_{n}\right), \quad \zeta_{b}=c_{b} /\left(2 m_{b} \omega_{n}\right), \\
\mu_{b}=m_{b} / M, \quad \eta=\frac{m_{b} d}{m_{d} e}
\end{gathered}
$$

In Eq. (1), ( )' indicates differentiation with respect to $\tau=\tau \omega_{n}$. It is worth noting that $\Omega$ indicates the ratio of the rotational speed to the natural frequency of the system and $\eta$ the ratio of the eccentricity of the ball to that of the disk.

\section{Equilibrium Positions}

The first step in analyzing a nonlinear system is to identify the equilibrium positions. The equilibrium positions, denoted by $\tilde{x}, \tilde{y}$, and $\widetilde{\beta}$, can be deduced from Eq. (1) by suppressing the time derivatives and solving the resulting algebraic equations,

$$
\left\{\begin{array}{l}
\left(1-\Omega^{2}\right) \tilde{x}-2 \zeta \Omega \tilde{y}-\mu_{b} \Omega^{2} \cos \tilde{\beta}-\mu_{b} \Omega^{2} / \eta=0 \\
2 \zeta \Omega \tilde{x}+\left(1-\Omega^{2}\right) \tilde{y}-\mu_{b} \Omega^{2} \sin \widetilde{\beta}=0 \\
\tilde{x} \sin \widetilde{\beta}-\tilde{y} \cos \widetilde{\beta}=0
\end{array}\right.
$$

To solve Eq. (3), we introduce the polar coordinates

$$
\tilde{x}=\tilde{r} \cos \tilde{\theta}, \quad \tilde{y}=\tilde{r} \sin \tilde{\theta} .
$$

Here $\tilde{r}$ denotes the residual vibration of the rotating disk. Then Eq. 3.3 can be rewritten as

$$
\tilde{r} \sin (\tilde{\beta}-\tilde{\theta})=0 .
$$

The above equation has three solutions: $\widetilde{\beta}=\widetilde{\theta}, \widetilde{\beta}=\widetilde{\theta}+\pi$, and $\widetilde{r}=0$. Equilibrium positions associated with these three cases are discussed separately below.

(i) $\widetilde{\beta}=\tilde{\theta}$

Substitution of $\widetilde{\beta}=\tilde{\theta}$ into Eqs. 3.1 and 3.2 yields two solutions for $\widetilde{r}$, which are referred to as $\widetilde{r}_{11}$ and $\widetilde{r}_{12}$ and expressed as

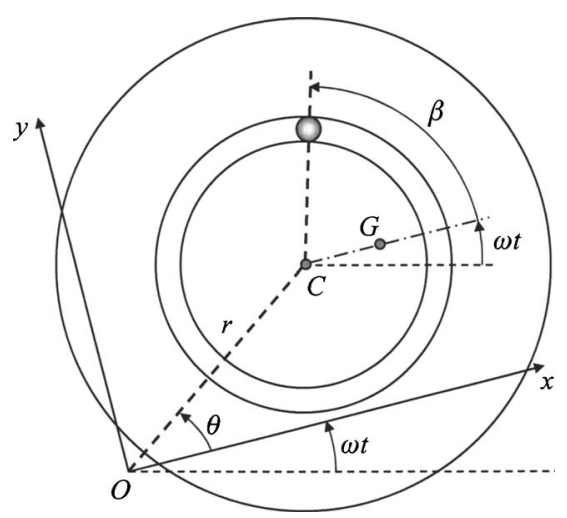

Fig. 2 Rotating coordinates system

$$
\left.\begin{array}{l}
\tilde{r}_{11} \\
\tilde{r}_{12}
\end{array}\right\}=\mathcal{K}\left[\eta\left(1-\Omega^{2}\right) \pm \sqrt{\mathcal{D}}\right]
$$

where

$$
\mathcal{K}=\frac{\mu_{b} \Omega^{2}}{\eta\left[\left(1-\Omega^{2}\right)^{2}+4 \zeta^{2} \Omega^{2}\right]} \quad \text { and } \quad \mathcal{D}=\left(1-\Omega^{2}\right)^{2}+4\left(1-\eta^{2}\right) \zeta^{2} \Omega^{2} \text {. }
$$

The corresponding $\tilde{\theta}$ is determined by

$$
\tilde{\theta}=\tan ^{-1} \frac{-2 \zeta \Omega \widetilde{r}}{\left(1-\Omega^{2}\right) \tilde{r}-\mu_{b} \Omega^{2}} .
$$

Note that $\tilde{r}$ should be non-negative. The conditions under which $\tilde{r} \geqslant 0$ are derived below for the cases $\eta<1$ and $\eta>1$, respectively. When $\eta<1$, as can be seen from Eqs. (4) and (5), $\widetilde{r}_{11}>0$ and $\widetilde{r}_{12}<0$. Therefore, $\widetilde{r}_{12}$ is not a possible equilibrium position for $\eta<1$. Then consider the case $\eta>1$. In this case, Eq. (5) indicates that

$$
\begin{cases}\mathcal{D}<0 & \text { for } \Omega_{1}<\Omega<\Omega_{2} \\ \mathcal{D}>0 & \text { otherwise }\end{cases}
$$

where

$$
\begin{gathered}
\Omega_{1}=\sqrt{g-\sqrt{g^{2}-1}}, \quad \Omega_{2}=\sqrt{g+\sqrt{g^{2}-1}}, \quad \text { and } \\
g=1+2\left(\eta^{2}-1\right) \zeta^{2} .
\end{gathered}
$$

Note that $\Omega_{1}<1<\Omega_{2}$ for $\eta>1$ and $\zeta \neq 0$. Equation (7) implies that $\widetilde{r}_{11}$ and $\widetilde{r}_{12}$ are complex in the region $\Omega_{1}<\Omega<\Omega_{2}$. As can be seen from Eq. (5), $\sqrt{\mathcal{D}}<\left|\eta\left(1-\Omega^{2}\right)\right|$ when $\mathcal{D}>0$ and $\eta>1$. In summary, for $\eta>1$,

$$
\begin{cases}\widetilde{r}_{11}, \widetilde{r}_{12}>0 & \text { for } 0<\Omega<\Omega_{1} \\ \widetilde{r}_{11}, \widetilde{r}_{12} \in \mathrm{C} & \text { for } \Omega_{1}<\Omega<\Omega_{2} \\ \widetilde{r}_{11}, \widetilde{r}_{12}<0 & \text { for } \Omega_{2}<\Omega\end{cases}
$$

The above results indicate that the ball cannot stay at the position $\beta=\theta$ when $\eta>1$ and $\Omega>\Omega_{1}$.

(ii) $\widetilde{\beta}=\tilde{\theta}+\pi$

Through a procedure similar to that in the previous case, we obtain two equilibrium positions, denoted by $\widetilde{r}_{21}$ and $\widetilde{r}_{22}$, as

$$
\left.\begin{array}{l}
\tilde{r}_{21} \\
\tilde{r}_{22}
\end{array}\right\}=\mathcal{K}\left[-\eta\left(1-\Omega^{2}\right) \pm \sqrt{\mathcal{D}}\right]
$$

where $\mathcal{K}$ and $\mathcal{D}$ are defined by Eq. (5). Then we proceed to determine the conditions under which $\tilde{r}$ is non-negative. When $\eta$ $<1$, it is clear that $\widetilde{r}_{22}<0$ but $\widetilde{r}_{21}>0$. On the other hand, when $\eta>1, \widetilde{r}_{21}, \widetilde{r}_{22}<0$ for $\Omega<\Omega_{1} ; \widetilde{r}_{21}, \widetilde{r}_{22}>0$ for $\Omega>\Omega_{2}$, where $\Omega_{1}$ 
Table 1 Equilibrium positions and their stability

\begin{tabular}{|c|c|c|c|c|c|}
\hline \multicolumn{2}{|c|}{$\eta<1$} & \multicolumn{2}{|c|}{$\eta=1$} & \multicolumn{2}{c|}{$\eta>1$} \\
\hline$\Omega<1$ & $\Omega>1$ & $\Omega<1$ & $\Omega>1$ & $\Omega<\Omega_{1}<1$ & $1<\Omega_{2}<\Omega$ \\
\hline$\tilde{r}=\tilde{r}_{11}$ & $\tilde{r}=\llbracket \tilde{r}_{11} \rrbracket$ & $\tilde{r}=0$ & $\tilde{r}=\llbracket 0 \rrbracket$ & $\tilde{r}=\tilde{r}_{11}$ & $\tilde{r}=\left[\tilde{r}_{21}\right.$ \\
or & or & or & or & or & or \\
$\tilde{r}_{21}$ & {$\left[\tilde{r}_{21}\right.$} & $\tilde{r}_{11}$ & $\tilde{r}_{21}$ & $\tilde{r}_{12}$ & $\llbracket \tilde{r}_{22} \rrbracket$ \\
\hline
\end{tabular}

and $\Omega_{2}$ are defined by Eq. (8). The position of the disk center $C$ relative to the $x$-axis is determined by

$$
\tilde{\theta}=\tan ^{-1} \frac{-2 \zeta \Omega \widetilde{r}}{\left(1-\Omega^{2}\right) \widetilde{r}+\mu_{b} \Omega^{2}} .
$$

(iii) $\tilde{r}=0$

In this case the system is perfectly balanced and has no residual vibration. Substituting $\widetilde{r}=0$ into Eq. (3) gives that $\beta=\pi$ and $\eta$ $=1$. In other words, perfect balancing is possible when the ball has the same eccentricity as the disk and is located on the opposite side of the diameter passing through the mass center of the disk.

The above analysis indicates that $\eta$ and $\Omega$ are two major factors affecting the equilibrium positions of the system. Possible equilibrium positions for the cases $\eta<1, \eta=1$, and $\eta>1$ are summarized in Table 1.

\section{Stability}

The behavior of the system depends on the stability of the equilibrium states. The stability of an equilibrium state can be determined by the eigenvalues of the linearized equation around the equilibrium state. The eigenvalues are roots of the corresponding characteristic equation as follows.

$$
p(s)=a_{0} s^{6}+a_{1} s^{5}+a_{2} s^{4}+a_{3} s^{3}+a_{4} s^{2}+a_{5} s+a_{6}=0,
$$

where the coefficients $a_{i}$, which are functions of the equilibrium positions, are given explicitly in the Appendix. According to Routh's criterion, information about stability can be obtained directly from the coefficients of the characteristic equation [8]. As can be seen from Eq. (A1) in the Appendix, $a_{0}$ is positive. In this case, an equilibrium state is unstable if any coefficient from $a_{1}$ to $a_{6}$ is negative. This result is used in the subsequent analysis to show that some equilibrium states are unstable under certain conditions. On the other hand, the necessary and sufficient condition for an equilibrium state to be asymptotically stable is that all the elements of the first column of the Routh array are of the same sign. The first column of the Routh array is tabulated in the Appendix. By using Routh's criterion we can investigate the stability of the equilibrium states analytically. The results are listed below.

(S1) The equilibrium state $\widetilde{r}=\widetilde{r}_{12}(\widetilde{\beta}=\widetilde{\theta})$ is unstable.

Substitution of the value of $\tilde{r}_{12}$ from Eq. (4) and $\tilde{\beta}=\tilde{\theta}$ into Eq. (A7) gives $a_{6}=-\left(\mu_{b} \Omega^{4} \sqrt{\mathcal{D}}\right) / \eta<0$. Therefore, $\tilde{r}=\widetilde{r}_{12}(\widetilde{\beta}=\tilde{\theta})$ is unstable.

(S2) The equilibrium state $\tilde{r}=\widetilde{r}_{21}(\tilde{\beta}=\tilde{\theta}+\pi)$ is unstable.

Substituting the value of $\widetilde{r}_{21}$ from Eq. (9) and $\widetilde{\beta}=\tilde{\theta}+\pi$ into Eq. (A7) yields $a_{6}=-\left(\mu_{b} \Omega^{4} \sqrt{\mathcal{D}}\right) / \eta<0$. Therefore $\tilde{r}=\tilde{r}_{21}(\tilde{\beta}=\tilde{\theta}+\pi)$ is unstable.

(S3) The equilibrium state $\widetilde{r}=0$ is unstable for $\Omega<1$.

Perfect balancing is possible only if $\eta=1$ and $\widetilde{\beta}=\pi$. In this case, Eq. (A7) reduces to $a_{6}=\Omega^{4}\left(\Omega^{2}-1\right) \mu_{b}$. Since $a_{6}<0$ for $\Omega$ $<1, \tilde{r}=0$ is unstable for $\Omega<1$.

Then consider the special case $\zeta=0$ but $\zeta_{b} \neq 0$.

(S4) The equilibrium state $\tilde{r}=0$ is unstable if $\zeta=0$ and $\zeta_{b} \neq 0$.
Substitution of $\tilde{r}=0$ and $\zeta=0$ into Eq. (A13) yields $A_{6}$ $=128 \Omega^{2}\left(1-\Omega^{4}\right) \zeta_{b}^{5} \mu_{b}^{3}$. It is obvious that, $A_{6}<0$ for $\Omega>1$, which in turn implies that $\widetilde{r}=0$ is unstable. Combination with (S3) leads to the conclusion that $\tilde{r}=0$ is unstable.

(S5) When $\zeta=0$ and $\zeta_{b} \neq 0$, the equilibrium state $\widetilde{r}=\widetilde{r}_{11}(\widetilde{\beta}=\widetilde{\theta})$ is stable for $\Omega<1$, and unstable for $\Omega>1$.

In this case, as can be seen from Eqs. (A8) to (A14), all the elements except $A_{6}$ of the first column of the Routh array are positive. In addition, $A_{6}$ can be expressed as

$$
A_{6}=8\left(1-\Omega^{2}\right) \zeta_{b} \mu_{b} / A_{5}=\left\{\begin{array}{cc}
>0 & \Omega<1 \\
<0 & \Omega>1
\end{array}\right.
$$

Since $A_{i}$ are of the same sign for $\Omega<1, \widetilde{r}=\widetilde{r}_{11}$ is stable for $\Omega$ $<1$ according to Routh's criterion. In contrast, there are two changes in sign of $A_{i}$ for $\Omega>1$. Hence, $\widetilde{r}=\widetilde{r}_{11}$ is unstable for $\Omega$ $>1$.

(S6) When $\zeta=0$ and $\zeta_{b} \neq 0$, the equilibrium state $\tilde{r}=\tilde{r}_{22}(\tilde{\beta}=\tilde{\theta}$ $+\pi)$ is unstable.

When $\zeta=0, \widetilde{r}_{22}<0$ for $\Omega<1$ and $\widetilde{r}_{22}=[(\eta-1) / \eta]\left[\mu_{b} \Omega^{2} /\left(\Omega^{2}\right.\right.$ -1)] for $\Omega>1$. We only need to investigate the stability of $\tilde{r}$ $=\widetilde{r}_{22}$ in the region $\Omega>1$ and $\eta>1$, where $\widetilde{r}_{22}$ is positive. Substituting $\zeta=0, \widetilde{r}=\widetilde{r}_{22}$ and $\widetilde{\beta}=\tilde{\theta}+\pi$ into Eqs. (A12) and (A13) gives that $A_{5} \cdot A_{6}=8 \Omega^{2}\left(1-\Omega^{2}\right) \zeta_{b} \mu_{b}<0$ for $\Omega>1$. This result indicates that there is at least one change in sign of $A_{i}$ for $\Omega>1$, which proves (S6).

Table 1 further summarizes the stability property of the equilibrium states. The unstable equilibrium states as shown in $(\mathrm{S} 1)-(\mathrm{S} 3)$ are enclosed by $\square$; the unstable equilibrium states when $\zeta=0$ but $\zeta_{b} \neq 0$, as shown in (S4)-(S6), are enclosed by [ ]. It is worth noting that there is at most one stable equilibrium position at a rotating speed. The equilibrium state $\left(\widetilde{r}=\widetilde{r}_{21}\right.$ and $\left.\widetilde{\beta}=\tilde{\theta}+\pi\right)$ is unstable. Furthermore, when $\zeta=0$ but $\zeta_{b} \neq 0, \widetilde{r}_{11}$ is stable for $\Omega$ $<1$ while no stable equilibrium state exists for $\Omega>1$.

\section{Conclusion}

Ball-type automatic balancers are used widely in the optical disk drive industry to suppress the vibrations induced by an unbalanced optical disk. We have investigated the stability property of a system representative of an optical disk drive equipped with a ball-type automatic balancer and a suspension system. The analytical nature of our investigation has yielded unique understanding of the stability property of the system and insights into the conditions under which perfect balancing can be achieved. Let $\Omega$ denote the ratio of the rotational speed of the disk to the natural frequency of the system. The results of the analysis can be summarized as follows.

(1) There is at most one stable equilibrium position at a rotating speed.

(2) Perfect balancing cannot be achieved physically if the suspension system is undamped.

(3) The perfect balancing position is unstable for $\Omega<1$.

(4) No stable equilibrium position exists for $\Omega>1$ if the suspension system is undamped.

\section{Acknowledgment}

This work was supported by the National Science Council of the Republic of China under the Grant No. NSC93-2212-E-002066.

\section{Appendix}

The coefficients of the characteristic polynomial are given below:

$$
a_{0}=1-\mu_{b}>0
$$




$$
\begin{gathered}
a_{1}=2\left[\zeta_{b}+\zeta\left(2-\mu_{b}\right)\right] \\
a_{2}=\left(1+\Omega^{2}\right)\left(2-\mu_{b}\right)+8 \zeta \zeta_{b}+\tilde{r} \Omega^{2} \cos (\tilde{\beta}-\tilde{\theta})+4 \zeta^{2} \\
a_{3}=4\left[\left(1+2 \zeta^{2}+\Omega^{2}\right) \zeta_{b}+\zeta\left(1+\Omega^{2}+\widetilde{r} \Omega^{2} \cos (\tilde{\beta}-\tilde{\theta})+\Omega^{2} \mu_{b}\right)\right] \\
a_{4}=\left(1-\Omega^{2}\right)^{2}+4 \zeta^{2} \Omega^{2}+2 \widetilde{r} \Omega^{2} \cos (\tilde{\beta}-\tilde{\theta})\left(1+2 \zeta^{2}+\Omega^{2}\right) \\
+8 \zeta\left(1+\Omega^{2}\right) \zeta_{b}+\Omega^{2}\left(6+\Omega^{2}\right) \mu_{b} \\
a_{5}=2\left\{\left[\left(1-\Omega^{2}\right)^{2}+4 \zeta^{2} \Omega^{2}\right] \zeta_{b}+\zeta \Omega^{2}\left[2 \widetilde{r}\left(1+\Omega^{2}\right) \cos (\widetilde{\beta}-\tilde{\theta})\right.\right. \\
\left.\left.+3 \Omega^{2} \mu_{b}\right]\right\} \\
a_{6}=\tilde{r} \Omega^{2}\left[\left(1-\Omega^{2}\right)^{2}+4 \zeta^{2} \Omega^{2}\right] \cos (\tilde{\beta}-\tilde{\theta})+\Omega^{4}\left(\Omega^{2}-1\right) \mu_{b}
\end{gathered}
$$

Let $A_{1}-A_{7}$ denote the elements of the first column of the Routh array. $A_{i}$ can be expressed as

$$
\begin{gathered}
A_{1}=a_{0} \\
A_{2}=a_{1} \\
A_{3}=\left(a_{1} a_{2}-a_{0} a_{3}\right) / a_{1}
\end{gathered}
$$

$$
\begin{gathered}
A_{4}=\left(A_{3} a_{3}-a_{1} B\right) / A_{3} \\
A_{5}=\left(A_{4} B-A_{3} D\right) / A_{4} \\
A_{6}=\left(A_{5} D-A_{4} a_{6}\right) / A_{5} \\
A_{7}=a_{6},
\end{gathered}
$$

where $B=\left(a_{1} a_{4}-a_{0} a_{5}\right) / a_{1}$ and $D=\left(A_{3} a_{5}-a_{1} a_{6}\right) / A_{3}$.

\section{References}

[1] Alexander, J. D., 1964, "An Automatic Dynamic Balancer," Proceedings for the Second Southeastern Conference, 2, pp. 415-426.

[2] Cade, J. W., 1965, "Self-compensating Balancing in Rotating Mechanicsm," Des. News, pp. 234-239.

[3] Bövik, P., and Högfors, C., 1986, "Autobalancing of Rotors," J. Sound Vib., 111(3), pp. 429-440.

[4] Rajalingham, C., Bhat, R. B., and Rakheja, S., 1998, "Automatic Balancing of Flexible Vertical Rotors Using a Guided Ball,” Int. J. Mech. Sci., 40(9), pp. $825-834$.

[5] Lee, J., and Moorhem, W. K. V., 1996, "Analytical and Experimental Analysis of a Self-Compensating Dynamic Balancer in a Rotating Mechanism," ASME J. Dyn. Syst., Meas., Control, 118, pp. 468-475.

[6] Chung, J., and Ro. D. S., 1999, "Dynamic Analysis of An Automatic Dynamic Balancer for Rotating Mechanisms," J. Sound Vib., 1999, pp. 1035-1056.

[7] Kang, J.-R., Chao, C.-P., Huang, C.-L., and Sung, C.-K., 2001, "The Dynamics of a Ball-Type Balancer System Equipped with a Pair of Free-Moving Balancing Masses," ASME J. Vibr. Acoust., 123, pp. 456-465.

[8] Ogata, K., 1970, Modern Control Engineering, Prentice-Hall, Inc., New Jersey. 